To the Editors:

\title{
Possible familial gestational spontaneous ovarian hyperstimulation syndrome due to mutation of FSH receptors (FGSOHS)
}

The ovarian hyperstimulation syndrome (OHSS) most often occurs as an iatrogenic complication following ovulation induction. Overproduction of endogenous human chorionic gonadotrophin (hCG) during pregnancy has been associated with spontaneous ovarian hyperstimulation syndrome [1]. Familial gestational spontaneous ovarian hyperstimulation syndrome (FGSOHS) due to mutation of FSH receptors has been described in a recent report, in which the familial pattern suggested a genetic cause. A heterozygous mutation in 
FSH receptors was identified in affected family members. Few mutations in FSH receptors have been reported but only one resulting in a gain of function. This mutation broadens the specificity of receptors, so that it responds to another ligand hCG $[1,2]$. Mutant FSH receptors lead to hypersensitivity to hCG. The onset and evolution of the syndrome coincides with the usual gestational time course fluctuation in hCG levels [3].

The proband, a 26-year old woman, presented in her second pregnancy with clinical features suggestive of OHSS. The first pregnancy had also been affected by the same condition, and had been terminated. The second pregnancy was spontaneously conceived. The patient had increasing abdominal pain, nausea and vomiting associated with abdominal distention at a period of amenorrhoea of 9 weeks. Ultrasound scanning showed a multieocular mass, measuring $10 \mathrm{~cm} \times 11 \mathrm{~cm}$ in both ovaries. Serum hCG levels were within the normal range for the period of gestation. She was managed conservatively with continued surveillance for complications. Subsequently, she underwent elective caesarian section at 38 weeks and delivered a normal baby. Ovarian biopsy was performed at the time of delivery and histology was compatible with OHS and excluded malignancy. Pedigree analysis showed that her two elder sisters had also had clinical features suggestive of OHSS in their pregnancies with no history of ovulation induction.

Although the definitive diagnosis of FGSOHS should be made by analysis of FSH receptors, in the absence of facilities to analyse mutant FSH receptors, our diagnosis was based on clinical features and a highly suggestive family history. Little is known about the pathophysiology of the FGSOHS [4,5]. The syndrome has a range of severity from mild to severe forms with the massive bilateral ovarian enlargement leading to life threatening situations requiring termination of the on going pregnancy [4].

\section{References}

1. Ursula BH. The pathogenesis of OHS. New England Journal of Medicine 2003; 349: 729-32 .

2. Claudine V, Etal MD. A chorionic Gonadotropin-sensitive mutation in the follicle-stimulating hormone receptor as a cause of familial gestational spontaneous ovarian hyperstimulation syndrome. New England Journal of Medicine 2003; 349: 753-9.

3. Elchalal U, Schenker JG. The pathophysiology of ovarian hyperstimulation syndrome - views and ideas. Human Reproduction 1997; 12: 1129-37.

4. Ludwig M, Gembruch U, Bauer O, Diedrich K. Ovarian hyperstimulation syndrome (OHSS) in a spontaneous pregnancy with fetal and placental triploidy: information about the general pathophysiology of OHSS. Human Reproduction 1998; 13: 2082-7.

5. Themmen APN, Huhtaniemi IT. Mutations of gonadotrophins and gonadotrophin receptors: elucidating the physiology and pathophysiology of pituitary-gonadal function. Endocrine Review 2000; 21: 551-83.

\section{L W Dasanayake, K Linganathan, P S Hettipathirana, G A Ranathunga and D Warnakulasuriya}

Castle Street Hospital for Women, Colombo 8, Sri Lanka.

Correspondence: DLWD, e-mail: <lanka.dasanayake@yahoo.com>. Received 28 February 2008 and revised version accepted 15 December 2008. Competing interests: none declared. 